Research Article

\title{
Intelligent Recognition Method of Turning Tool Wear State Based on Information Fusion Technology and BP Neural Network
}

\author{
Yanwei Xu $\mathbb{D}^{1,2}$ Lin Gui, ${ }^{1}$ and Tancheng $X^{1,2}$ \\ ${ }^{1}$ School of Mechatronics Engineering, Henan University of Science and Technology, Luoyang 471003, China \\ ${ }^{2}$ Intelligent Numerical Control Equipment Engineering Laboratory of Henan Province, Luoyang 471003, China \\ Correspondence should be addressed to Yanwei Xu; xuyanweiluoyang@163.com
}

Received 28 April 2021; Revised 11 June 2021; Accepted 10 July 2021; Published 20 July 2021

Academic Editor: Zi-Qiang Lang

Copyright (c) 2021 Yanwei Xu et al. This is an open access article distributed under the Creative Commons Attribution License, which permits unrestricted use, distribution, and reproduction in any medium, provided the original work is properly cited.

The multi-information data acquisition system of tool wear condition of CNC lathe is built by acquiring the acoustic emission and vibration acceleration signals. The data of acoustic emission and vibration acceleration signals during the process of CNC machine tool processing under the conditions of different tool wear degrees and different cutting conditions are acquired and analyzed using the orthogonal experimental method. The optimum characteristic frequency band of acoustic emission and vibration acceleration signals was extracted by the wavelet envelope decomposition method so as to recognize tool wear condition as the characteristic parameters. The characteristic information of acoustic emission and vibration acceleration signals during the process of $\mathrm{CNC}$ machine tool processing was fused. In addition, the intelligent recognition of tool wear condition during the process of machine tool processing was researched.

\section{Introduction}

Tool wear and breakage will occur in the process of CNC machine tool cutting, which will directly affect the machining accuracy and surface quality of the workpiece. Tool wear and breakage will also directly affect the machining efficiency and cutting stability of the whole manufacturing equipment system. According to statistics, the failure shutdown rate caused by tool failure accounts for about $22.4 \%$ of the total failure shutdown rate of CNC machine tools. In the actual production process [1], there is a highly nonlinear relationship between the influencing factors and the tool wear [2], so it is difficult to judge the tool wear accurately by the manual method. In order to ensure the accuracy of machining, it is necessary to replace the tool before the tool is severely worn, so it is necessary to replace the tool frequently, but the frequent replacement of the tool reduces the efficiency of production and improves the cost of machining. If the serious situation of tool wear and damage is not found in time or the tool is not replaced in time, the processing process may be interrupted, and the workpiece may be scrapped. By monitoring and identifying the tool wear status, the tool can be replaced at the right time. Monitoring, identifying the tool wear status, and timely replacing the tool not only can ensure the machining accuracy but also can improve the tool utilization and reduce the production cost. Replacing the cutting tool at the right time can avoid the scrapping of the workpiece and the failure of the machine tool caused by the failure of the cutting tool.

In recent years, many experts and scholars have studied the methods to identify the tool wear state [3-9], and their research has promoted the development of tool wear state identification technology. However, many experts and scholars study the tool wear state identification method based on the information provided by a single sensor, and it is difficult to ensure that a single sensor provides correct and comprehensive information. Information fusion technology is a comprehensive decision-making process to accurately estimate the measurement results of multiple sensors, so as to comprehensively and timely evaluate the situation, threat, and importance. Therefore, information fusion technology has the advantages of high fault tolerance, complementarity, economy, and being real time [10-17]. In recent years, information fusion technology has been applied more and 
more in the field of tool wear state recognition, but there is no unified standard [18-22].

At present, the common methods of multi-sensor data fusion can be summarized as random and artificial intelligence. Artificial intelligence includes fuzzy logic reasoning, neural network, and so on. The representation and processing of information by fuzzy logic reasoning is closer to the way of human thinking, but there are many subjective factors in the description of information, so the representation and processing of information by fuzzy logic reasoning lacks objectivity. Neural network has strong fault tolerance, self-learning, self-organization, and self-adaptive ability. It can simulate complex nonlinear mapping and meet the requirements of multi-sensor data fusion technology. In this paper, BP neural network technology is used to fuse the acoustic emission (AE) signal of tool wear and the characteristic information of vibration signal in the process of numerical control cutting, so as to study the intelligent recognition technology of tool wear state in the process of numerical control turning.

\section{Acquisition System of Tool Wear Condition}

2.1. The Structure of the System. The acquisition system of tool wear condition is composed of a CNC lathe, workpiece, turning tool, AE sensor, vibration sensor, amplifier, signal conditioner, data acquisition card, and computer (the structure diagram of the system is shown in Figure 1).

The AE sensor and vibration sensor are placed in the tool handle. The signal detected by the sensor is processed by the preamplifier and signal conditioner and then transmitted to the computer by data acquisition card for analysis and processing. Figure 2 shows the physical diagram of the multi-information data acquisition system of tool wear condition of the CNC lathe, which is composed of a CKJ6152 CNC lathe, PCI- 8 acoustic emission instrument, WD/FM01 broadband differential AE sensor, LC0151T acceleration sensor, LC0201-5 signal conditioner matched with acceleration sensor, and PCI8510 data acquisition card. The signal-to-noise ratio of the acoustic emission instrument is 4.5 , the frequency range is $1 \mathrm{kHz}-3 \mathrm{MHz}$, and the maximum sampling frequency is $10 \mathrm{~m} / \mathrm{s}$. The AE sensor collects the voltage signal, the measurement range is $1 \mathrm{kHz}-1 \mathrm{MHz}$, and the resonant frequency is $531.25 \mathrm{kHz}$. The sensitivity of the acceleration sensor is $150 \mathrm{mV} / \mathrm{g}$, the measurement range is $33 \mathrm{~g}$, the resolution is $40 \mathrm{kHz}$, the resonant frequency is $0.0002 \mathrm{~g}$, and the frequency range is $0.7 \mathrm{~Hz}-13 \mathrm{kHz}$. And the 8 -channel data acquisition card with the speed of $500 \mathrm{k} / \mathrm{s}$ is adopted is $500 \mathrm{k} / \mathrm{s}$.

2.2. The Location of Sensors. The vibration sensor and $\mathrm{AE}$ sensor are fixed on the new turning tool of CNC lathe according to different directions and distances. The material of the turning tool is YT15. The turning test of 45\# steel bar is carried out under the conditions of spindle speed $n=800 \mathrm{r} / \mathrm{min}$, feed rate $f=40 \mathrm{~mm} / \mathrm{min}$, and back engagement ap $=0.5 \mathrm{~mm}$. The signals of vibration sensor and $\mathrm{AE}$ sensor are collected and analyzed to determine the reasonable fixed position of the sensor.

As shown in Figure 3, the $\mathrm{AE}$ sensor is tested in five installation positions. Position 1 is $15 \mathrm{~cm}$ from the tip at the rear of the left side of the handle, position 2 is $4 \mathrm{~cm}$ from the tip at the front of the left side of the handle, position 3 is $2 \mathrm{~cm}$ from the tip at the right side of the handle, position 4 is $4 \mathrm{~cm}$ from the tip at the right side of the handle, and position 5 is $6 \mathrm{~cm}$ from the tip at the right side of the handle. We find the root-mean-square value of data collected by $\mathrm{AE}$ sensor and judge the strength of signal according to the value of rootmean-square value. The results show that the root-meansquare values of positions 3, 4, and 5 decrease in turn, while the root-mean-square values of positions 2 and 4 are basically the same, indicating that the signal strength is independent of direction, while position 1 has the smallest root-mean-square value. Therefore, the best installation position for the $\mathrm{AE}$ sensor is position 3 .

As shown in Figure 4, the acceleration sensor is tested in three different positions: position 1 is $4 \mathrm{~cm}$ away from the tip, position 2 is $8 \mathrm{~cm}$ away from the tip, position 3 is $12 \mathrm{~cm}$ away from the tip, and the three spatial positions are shown in $X, Y$, and $Z$ directions in Figure 4 . The results show that position 1 and $Z$ direction have the largest root-mean-square value, so position 1 and $Z$ direction are selected as the best installation position for acceleration sensor.

2.3. Dividing the Degree of Tool Wear. One kind of indexable cemented carbide tool CNMG120404-PM and 45\# steel bar are selected as test objects. And the parameters of the tool CNMG120404-PM are as follows: tool material YT15, tool rake angle $\gamma=11^{\circ}$, clearance angle $\alpha=10^{\circ}$, tool cutting edge angle $\mathrm{Kr}^{\prime}=95^{\circ}$, tool cutting edge inclination angle $\lambda=0^{\circ}$. Cutting tests are carried out according to engagement ranging from $0.3 \mathrm{~mm}$ to $1.0 \mathrm{~mm}$ to obtain tool test samples of different wear degrees.

During the turning process, the wear of the flank surface has a great influence on the quality of the workpiece, cutting force, and cutting temperature. Since the wear of the flank surface is easy to observe and measure [23], according to the international standard ISO 3685-1977, the wear band width VB measured at the middle part of the flank surface is selected as the test standard, and the tool wear state is divided into 4 wear degrees as shown in Table 1.

2.4. Orthogonal Experimental Method. Taking the tool wear degrees, spindle speed, feed rate, and cutting depth as the main factors, four kinds of blade samples with different wear degrees were selected, and 16 groups of turning experiments were carried out by the orthogonal experimental method. The AE signal and vibration signal were collected in each group of tests, and the influence degree of different factors on AE signal and vibration signal was analyzed. 


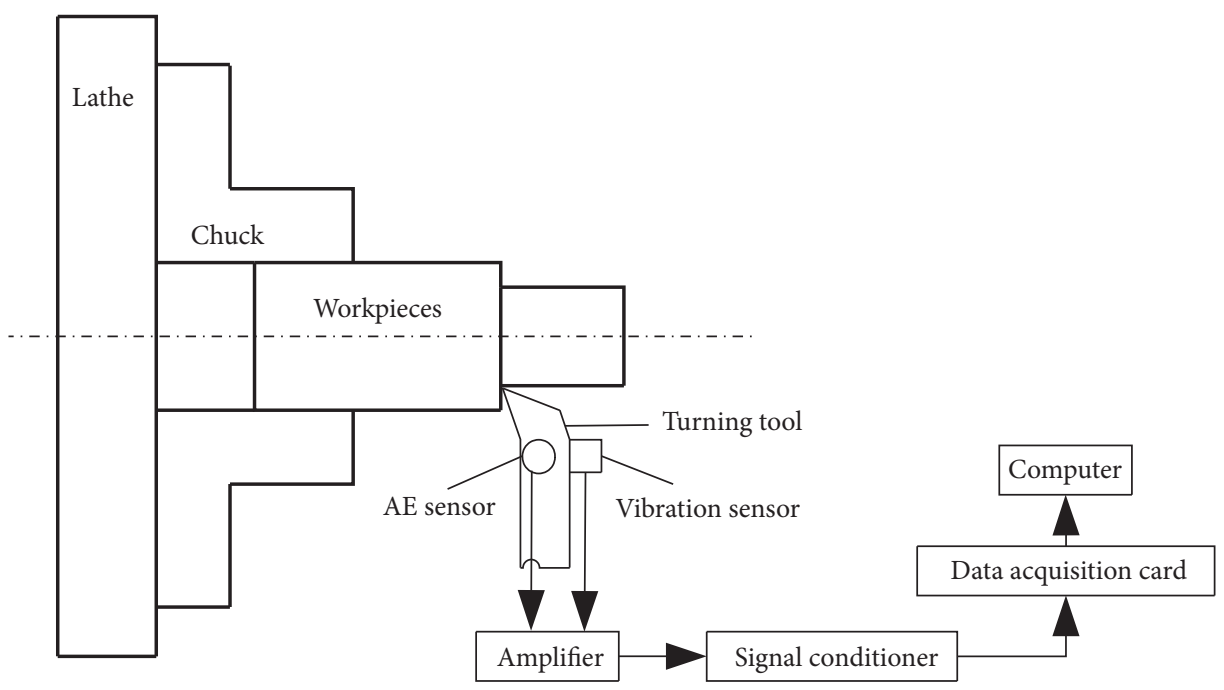

FIgURE 1: The structure diagram of the system.

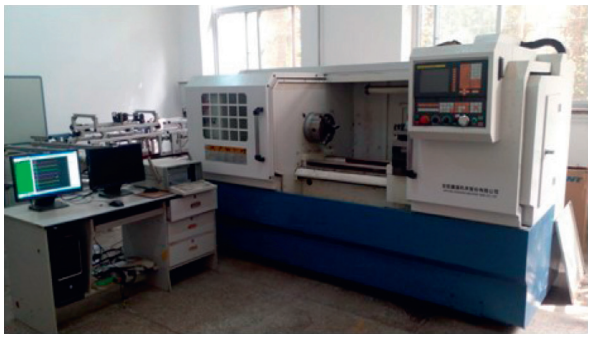

FIgURE 2: The physical diagram of the multi-information data acquisition system of tool wear condition of $\mathrm{CNC}$ lathe.

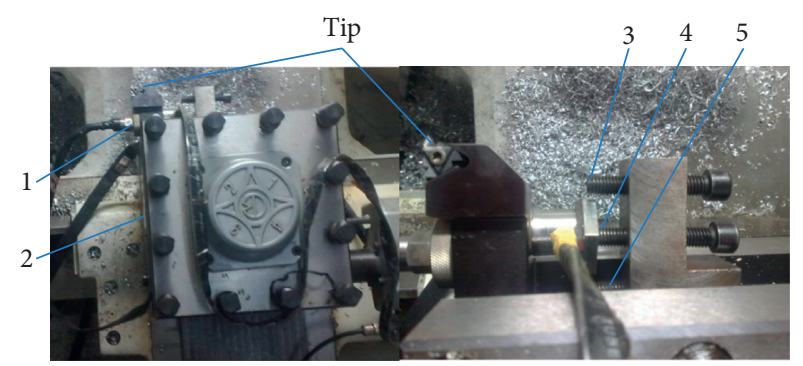

FIgURE 3: Installation position of AE sensor.

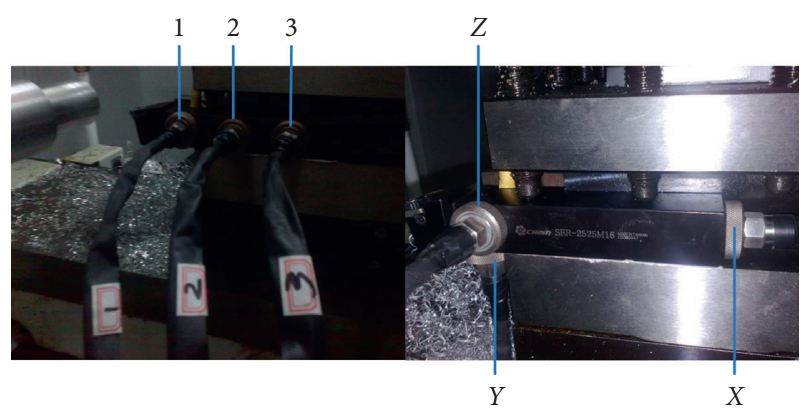

Figure 4: Installation position of acceleration sensor.
TABle 1: Tool wear degrees.

\begin{tabular}{lcccc}
\hline Classification & $\begin{array}{c}\text { Initial } \\
\text { wear }\end{array}$ & $\begin{array}{c}\text { Normal } \\
\text { wear }\end{array}$ & $\begin{array}{c}\text { Medium } \\
\text { wear }\end{array}$ & $\begin{array}{c}\text { Severe } \\
\text { wear }\end{array}$ \\
\hline $\begin{array}{l}\text { Degrees } \\
\text { VB values } \\
(\mathrm{mm})\end{array}$ & 1 & 2 & 3 & 4 \\
\hline
\end{tabular}

The test conditions are cutting with cutting fluid. The material processed is $45 \#$ steel bar, and the experimental time of each group is $30 \mathrm{~s}$.

Table 2 shows the experimental parameters and the data collected by the sensor, and Table 3 shows the analysis results of the experimental data using the mean method.

According to the analysis results in Table 3, for AE signal, the maximum range of wear degree is 1.9357 , the maximum range of spindle speed is 2.5541, the maximum range of feed speed is 0.3083 , and the maximum range of cutting depth is 0.5408 . Therefore, the spindle speed has the most significant effect on $\mathrm{AE}$ signal, followed by wear degree and cutting depth, and the feed speed has the least effect on AE signal. For vibration signal, the maximum range of wear degree is 0.5346 , the maximum range of spindle speed is 0.2944 , the maximum range of feed speed is 0.2650 , and the maximum range of cutting depth is 0.1615 . Therefore, the influence of wear degree on vibration signal is the most significant, followed by spindle speed and feed speed, and the influence of cutting depth on vibration signal is the least.

\section{Selection of Tool Wear Characteristics}

Acoustic emission and vibration signals are nonstationary random signals whose frequency and statistical characteristics vary with time. Spectrum analysis can analyze the characteristics of nonstationary random signals in a certain period. Wavelet packet analysis is a kind of signal processing technology, which concentrates the information energy, finds the order in the details, and selects the rules, and it can divide the frequency band into multiple levels and can adaptively select the corresponding frequency band according to the 
TABle 2: Experimental parameters and collected data.

\begin{tabular}{|c|c|c|c|c|c|c|}
\hline \multirow{2}{*}{$\begin{array}{l}\text { Serial } \\
\text { numbers }\end{array}$} & \multicolumn{4}{|c|}{ Experimental parameters } & \multicolumn{2}{|c|}{ Collected data } \\
\hline & Degrees of wear & $\begin{array}{l}\text { Spindle speed }(\mathrm{r} / \\
\min )\end{array}$ & $\begin{array}{l}\text { Feed rate }(\mathrm{mm} / \\
\text { min })\end{array}$ & $\begin{array}{l}\text { Cutting depth } \\
(\mathrm{mm})\end{array}$ & AE sensor $(\mathrm{V})$ & Vibration sensor $(\mathrm{V})$ \\
\hline 1 & 1 & 560 & 20 & 0.4 & 0.7522 & 0.1542 \\
\hline 2 & 1 & 800 & 30 & 0.6 & 1.4141 & 0.1732 \\
\hline 3 & 1 & 1120 & 40 & 0.8 & 2.0434 & 0.2119 \\
\hline 4 & 1 & 1600 & 50 & 1.0 & 3.1657 & 0.2183 \\
\hline 5 & 2 & 560 & 30 & 0.8 & 1.1045 & 0.3117 \\
\hline 6 & 2 & 800 & 20 & 1.0 & 1.9643 & 0.2944 \\
\hline 7 & 2 & 1120 & 50 & 0.4 & 1.7929 & 0.2357 \\
\hline 8 & 2 & 1600 & 40 & 0.6 & 3.9895 & 0.2698 \\
\hline 9 & 3 & 560 & 40 & 1.0 & 2.3615 & 0.4471 \\
\hline 10 & 3 & 800 & 50 & 0.8 & 3.3478 & 0.3960 \\
\hline 11 & 3 & 1120 & 20 & 0.6 & 4.4762 & 0.1940 \\
\hline 12 & 3 & 1600 & 30 & 0.4 & 4.9327 & 0.2112 \\
\hline 13 & 4 & 560 & 50 & 0.6 & 2.0609 & 1.2597 \\
\hline 14 & 4 & 800 & 40 & 0.4 & 2.2999 & 0.6498 \\
\hline 15 & 4 & 1120 & 30 & 1.0 & 4.0951 & 0.3535 \\
\hline 16 & 4 & 1600 & 20 & 0.8 & 4.4076 & 0.6331 \\
\hline
\end{tabular}

TABLe 3: Analysis result of experiment data.

\begin{tabular}{|c|c|c|c|c|c|}
\hline \multirow{2}{*}{\multicolumn{2}{|c|}{ Signal classification }} & \multicolumn{4}{|c|}{ Influence factors } \\
\hline & & Degrees of wear & Spindle speed & Feed rate & Cutting depth \\
\hline \multirow{5}{*}{ AE signal } & Mean 1 & 1.8439 & 1.5698 & 2.9001 & 2.4444 \\
\hline & Mean 2 & 2.2128 & 2.2565 & 2.8866 & 2.9852 \\
\hline & Mean 3 & 3.7796 & 3.1019 & 2.6736 & 2.7258 \\
\hline & Mean 4 & 3.2159 & 4.1239 & 2.5918 & 2.8967 \\
\hline & Range & 1.9357 & 2.5541 & 0.3083 & 0.5408 \\
\hline \multirow{5}{*}{ Vibration signal } & Mean 1 & 0.1894 & 0.5432 & 0.3189 & 0.3127 \\
\hline & Mean 2 & 0.2779 & 0.3784 & 0.2624 & 0.4742 \\
\hline & Mean 3 & 0.3121 & 0.2488 & 0.3947 & 0.3882 \\
\hline & Mean 4 & 0.7240 & 0.3331 & 0.5274 & 0.3283 \\
\hline & Range & 0.5346 & 0.2944 & 0.2650 & 0.1615 \\
\hline
\end{tabular}

characteristics of the analyzed signal, so that it can match the signal spectrum and improve the resolution of time-frequency, and it is widely used in engineering practice. In this paper, the wavelet packet decomposition method is used to decompose the test data, and the energy value of each frequency band signal is obtained. Then, according to the mean and range of each frequency band energy under different conditions, the relevant characteristic frequency band of acoustic emission and vibration signal is selected.

3.1. Selection of Vibration Signal Characteristics. The vibration signal of tool wear is mainly in the low-frequency band (no more than $8 \mathrm{kHz}$ ) [24]. Considering the integrity of the collected signal, the sensor with the frequency range of $0.7-13 \mathrm{kHz}$ and sampling speed of $250 \mathrm{k} / \mathrm{s}$ is selected to collect the signal, and the $\mathrm{db} 8$ is selected as the wavelet base to decompose the collected signal by 6 -level wavelet packet, and then the frequency difference of each frequency band is reduced to $1.95 \mathrm{kHz}$. The 6-level wavelet packet decomposition of medium wear tool vibration signal in 13-16 frequency bands is shown in Figure 5.

Since the effective frequency of the signal is not more than $13 \mathrm{kHz}$, only the first 16 of 64 bands $(0 \sim 31.2 \mathrm{kHz})$ are analyzed to avoid information redundancy. The energy changes of the first 16 frequency bands of vibration signals with different tool wear degrees are shown in Figure 6.

It can be seen from Figure 6 that the energy is mainly concentrated in P1, P2, P3, P4, P7, and P8 frequency bands. With the increase of tool wear degree, the energy increases significantly in P2, P4, P7, and P8 frequency bands. Since the effective frequency range of acceleration sensor is $0.7 \mathrm{~Hz} \sim 13 \mathrm{kHz}( \pm 10 \%)$, the P8 band is omitted. The characteristic frequency bands of vibration signal are P2, P4, and P7.

Then, the mean and range of energy values in P2, P4, and P7 frequency bands under different conditions are calculated by orthogonal table to determine the characteristic frequency band which can best reflect the wear degree and is least affected by external factors. Table 4 shows the mean and range of energy values of vibration signals in P2, P4, and P7 frequency bands obtained by using orthogonal test table.

According to Table 4, the wear degree has the greatest influence on the energy changes of P2, P4, and P7 bands, which is consistent with the analysis results in Table 3.

In $\mathrm{P} 2$ band $(1.95 \sim 3.9 \mathrm{kHz})$, the range of wear degree is 24.96, which is much larger than that of the other three 


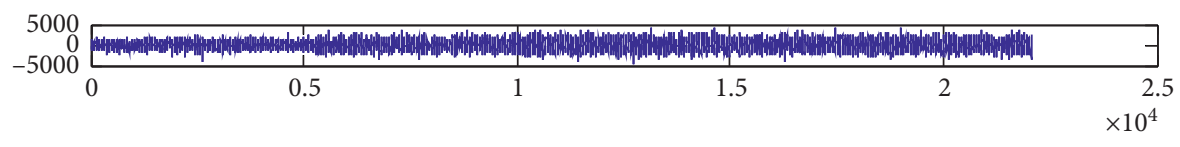

(a)

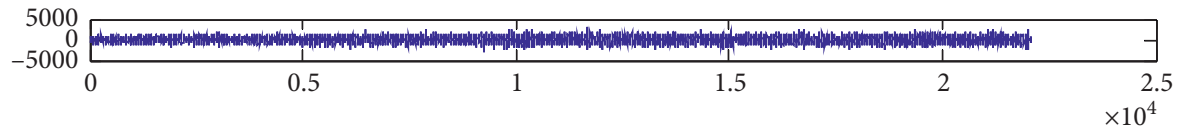

(b)

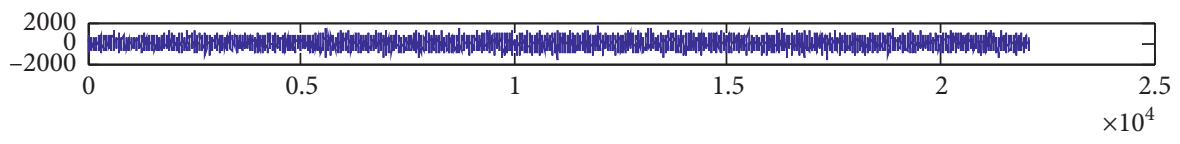

(c)

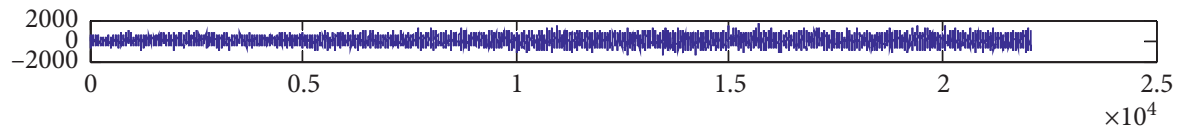

(d)

Figure 5: 6-level wavelet packet decomposition of medium wear tool vibration signal in 13-16 frequency bands. (a) P13. (b) P14. (c) P15. (d) P16.

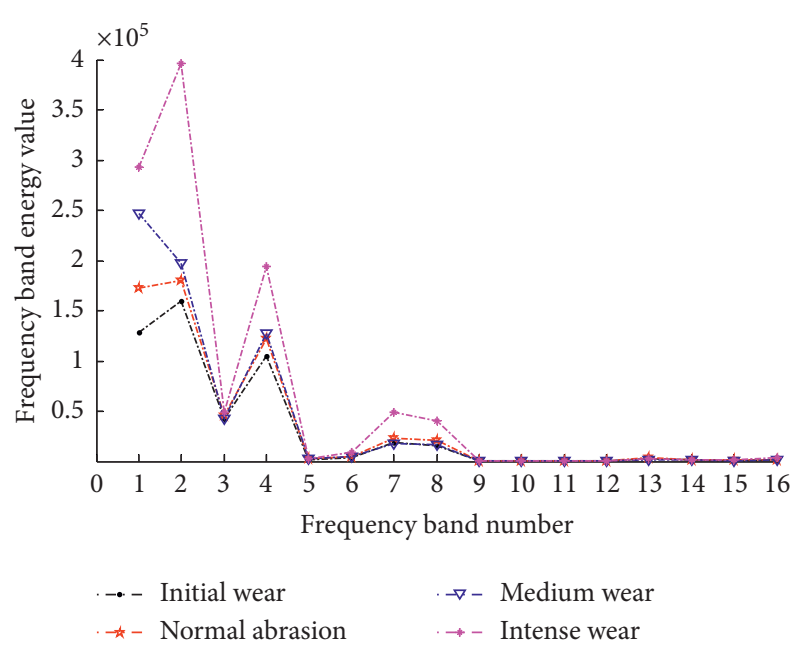

Figure 6: Energy value of vibration signal in different frequency bands.

factors, which are 3.35 times, 7.66 times, and 4.11 times that of spindle speed, feed speed, and cutting depth, respectively. The P2 frequency band is the most important one, and the three factors of cutting cannot be considered, so it is an ideal characteristic frequency band.

In $\mathrm{P} 4$ band $(5.85 \sim 7.8 \mathrm{kHz})$, the range of wear degree is 41.88, which is 2.18 times, 3.23 times, and 3.70 times that of the range of spindle speed, feed speed, and cutting depth, respectively. In the $\mathrm{P} 4$ frequency band, the wear degree has the greatest influence on the energy change, but the influence of the three cutting factors on the energy change is relatively small, so $\mathrm{P} 4$ can be selected as the characteristic frequency band.
The range of wear degree in $\mathrm{P} 7 \mathrm{band}(11.7 \sim 13.65 \mathrm{kHz})$ is 18.59 , which is 1.27 times, 1.44 times, and 2.02 times that of spindle speed, feed speed, and cutting depth, respectively. P7 band cannot be used as characteristic band.

Therefore, $\mathrm{P} 2$ is the best characteristic band of vibration signal, followed by $\mathrm{P} 4$.

3.2. Feature Selection of AE Signal. The acoustic emission signal of tool wear is an ultra-high frequency stress wave pulse signal with a frequency of more than $100 \mathrm{kHz}$ released when dislocation, crack propagation, and plastic deformation occur in the molecular lattice during metal processing [25]. This paper selects $100 \mathrm{kHz} 1 \mathrm{MHz}$ sensor, with a sampling frequency of $2 \mathrm{~m} / \mathrm{s}$, adopts 5-stage wavelet packet decomposition, and selects $\mathrm{dB} 8$ as wavelet base to reduce the frequency difference of each frequency band to $31.25 \mathrm{kHz}$. The wavelet packet decomposition of AE signal shows that the energy mainly exists in P1 P16 bands $(0 \sim 500 \mathrm{kHz})$, and there is almost no energy in P17 P32 bands, so only P1 P16 bands are analyzed. The energy changes of the first 16 bands of acoustic emission signals with different wear degree of the tool are shown in Figure 7.

It can be seen from Figure 7 that the energy is mainly concentrated in P1 P8 and P13 frequency bands. With the increase of tool wear degree, the energy increases significantly in P1, P2, P4, P7, and P13 frequency bands. Since the effective frequency band of $\mathrm{AE}$ signal is greater than $100 \mathrm{kHz}, \mathrm{P} 1$ and P2 frequency bands $(0 \sim 62.5 \mathrm{kHz})$ are omitted. The characteristic frequency bands of AE signal are P4, P7, and P13.

Then, the mean and range of energy values in $\mathrm{P} 4, \mathrm{P} 7$, and P13 frequency bands under different conditions are calculated by orthogonal table to determine the characteristic 
TABLE 4: Energy analysis results of P2, P4, and P7 frequency bands of vibration signal $\left(\times 10^{5}\right)$.

\begin{tabular}{|c|c|c|c|c|c|}
\hline \multirow{2}{*}{ Frequency band } & \multirow{2}{*}{ Mean and range } & \multicolumn{4}{|c|}{ Experimental factors } \\
\hline & & Degrees of wear & Spindle speed & Feed rate & Cutting depth \\
\hline \multirow{5}{*}{$\mathrm{P} 2$} & Mean 1 & 6.97 & 21.07 & 14.97 & 12.47 \\
\hline & Mean 2 & 11.97 & 13.63 & 15.46 & 18.54 \\
\hline & Mean 3 & 12.09 & 13.84 & 14.64 & 16.45 \\
\hline & Mean 4 & 31.93 & 14.43 & 17.9 & 15.51 \\
\hline & Range & 24.96 & 7.44 & 3.26 & 6.07 \\
\hline \multirow{5}{*}{$\mathrm{P} 4$} & Mean 1 & 7.66 & 33.08 & 18.14 & 20.67 \\
\hline & Mean 2 & 13.38 & 22.83 & 15.84 & 28.32 \\
\hline & Mean 3 & 16.33 & 13.84 & 24.12 & 20.92 \\
\hline & Mean 4 & 49.54 & 17.16 & 28.82 & 17.00 \\
\hline & Range & 41.88 & 19.24 & 12.98 & 11.32 \\
\hline \multirow{5}{*}{ P7 } & Mean 1 & 6.46 & 22.54 & 10.29 & 10.15 \\
\hline & Mean 2 & 10.28 & 11.91 & 8.42 & 19.37 \\
\hline & Mean 3 & 11.36 & 7.92 & 13.15 & 12.07 \\
\hline & Mean 4 & 25.05 & 10.77 & 21.29 & 11.55 \\
\hline & Range & 18.59 & 14.62 & 12.87 & 9.22 \\
\hline
\end{tabular}

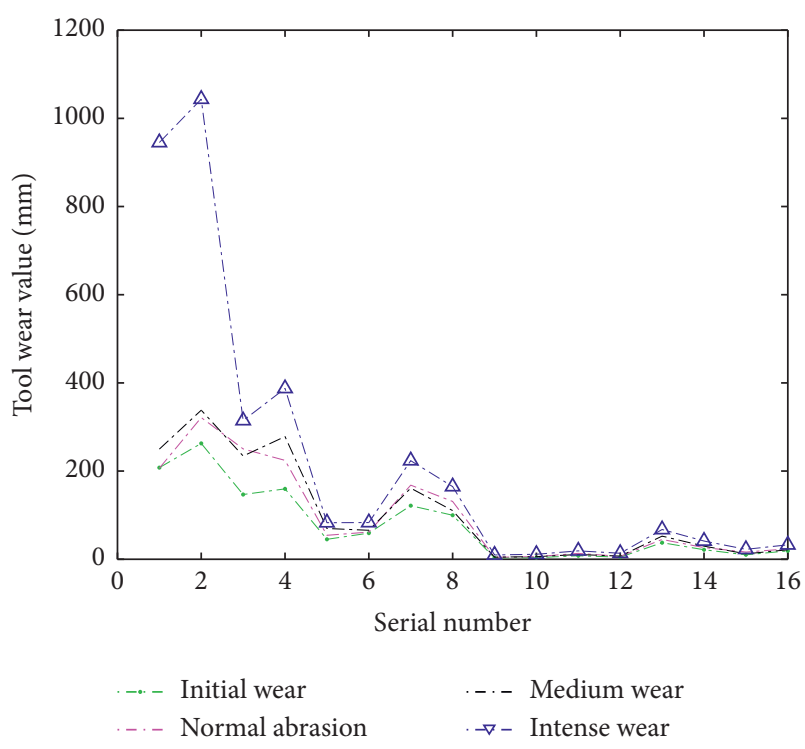

FIGURE 7: Energy value of AE signal in different frequency bands.

frequency band which can best reflect the wear degree and is least affected by external factors. Table 5 shows the mean value and range of energy value of vibration signal P4, P7, and P13 under different conditions.

It can be seen from Table 5 that the spindle speed has the greatest influence on the energy change of P4, P7, and P13 bands, which is consistent with the analysis results in Table 3.

In $\mathrm{P} 4$ frequency band $(93.75 \sim 125 \mathrm{kHz})$, the range of spindle speed is 1432.7 , the influence of tool wear degree is second only to spindle speed (range is 1116.6), and the influence of feed speed and cutting depth is relatively small (range is 568.6 and 692, respectively).

In P7 frequency band $(187.5 \sim 218.75 \mathrm{kHz})$ and P13 frequency band $(375 \sim 406.25 \mathrm{kHz})$, the influence of wear degree is the second, but the influence of wear degree is similar to that of feed speed and cutting depth, so it is not suitable to be used as characteristic frequency band.

Therefore, it is more suitable to select P4 band as the characteristic band of AE signal in tool wear detection. At the same time, considering that the acoustic emission signal characteristics in tool wear monitoring system are greatly affected by the spindle speed, in order to reduce the error rate of intelligent recognition, the spindle speed is also selected as the characteristic value of AE signal.

In view of the small influence of cutting depth and feed speed on vibration and acoustic emission signals, the shortterm experimental processing was carried out on the obtained worn blade samples by keeping the cutting depth and feed speed at the constant value and selecting the spindle speed randomly according to the orthogonal test speed. The acoustic emission and vibration signals of each blade during processing were collected, and the energy values of $\mathrm{P} 2$ and $\mathrm{P} 4$ frequency bands of vibration signals, the energy values of P4 frequency band of acoustic emission signals, and the corresponding spindle speed are extracted as the required characteristic parameters for the training and verification of the intelligent recognition model of tool wear state. The test parameters of this short-term experimental processing are as follows: cutting depth ap is $0.6 \mathrm{~mm}$, feed speed vf is $30 \mathrm{~mm} /$ min, spindle speed random, using cutting fluid, processing material 45\# steel bar, and each blade test time is $30 \mathrm{~s}$.

\section{Information Fusion Model Based on BP Neural Network}

The cutting process is a complex nonlinear stochastic process with many variables. The relationship between the tool wear state and the monitored signal is also highly nonlinear. Artificial intelligence algorithms such as support vector machine (SVM), fuzzy clustering analysis (FCA), and neural network (NN) are widely used in tool wear condition 
TABLE 5: Energy analysis results of P4, P7, and P13 frequency bands of AE signal.

\begin{tabular}{|c|c|c|c|c|c|}
\hline \multirow{2}{*}{ Frequency band } & \multirow{2}{*}{ Mean and range } & \multicolumn{4}{|c|}{ Experimental factors } \\
\hline & & Degrees of wear & Spindle speed & Feed rate & Cutting depth \\
\hline \multirow{5}{*}{ P4 } & Mean 1 & 1051.9 & 732.2 & 1821.5 & 1281.7 \\
\hline & Mean 2 & 1182.8 & 1159.8 & 1607.2 & 1754.3 \\
\hline & Mean 3 & 2157.1 & 2007.4 & 1306.6 & 1062.3 \\
\hline & Mean 4 & 1116.6 & 2164.9 & 1252.9 & 1618.9 \\
\hline & Range & 1105.2 & 1432.7 & 568.6 & 692 \\
\hline \multirow{5}{*}{ P7 } & Mean 1 & 372.3 & 138.6 & 439.5 & 376.7 \\
\hline & Mean 2 & 331.4 & 250.9 & 499 & 432.3 \\
\hline & Mean 3 & 484.3 & 493.7 & 397 & 434.1 \\
\hline & Mean 4 & 489.3 & 794.1 & 341.7 & 434.2 \\
\hline & Range & 157.9 & 655.5 & 157.3 & 57.5 \\
\hline \multirow{5}{*}{ P13 } & Mean 1 & 309.1 & 117.7 & 383.4 & 316.3 \\
\hline & Mean 2 & 255.8 & 209.1 & 422.5 & 354.5 \\
\hline & Mean 3 & 404.1 & 417.1 & 325.7 & 382.1 \\
\hline & Mean 4 & 445.0 & 670 & 282.4 & 361.1 \\
\hline & Range & 189.2 & 552.3 & 140.1 & 65.8 \\
\hline
\end{tabular}

monitoring and recognition. Support vector machine (SVM) is a pattern recognition method based on statistical learning theory and structural risk minimization principle. It takes into account the training error and generalization ability. Support vector machine (SVM) is a pattern recognition method based on statistical learning theory and structural risk minimization principle and taking into account the training error and generalization ability. SVM is widely used in pattern recognition and feature extraction such as small sample, high dimension, nonlinear, and local minimum. Fuzzy clustering analysis is an analysis method that establishes the fuzzy similarity relationship according to the characteristics, intimacy, and similarity of objective things based on the fuzzy mathematics and then classifies and identifies the fuzzy state of samples according to a certain membership degree, so as to cluster objective things. Fuzzy clustering analysis method is widely used in meteorological forecast, geology, agriculture, forestry monitoring, and other aspects. It is widely used in meteorological forecast, geology, agriculture, forestry monitoring, and other aspects. Artificial neural network is a nonlinear and adaptive information processing system composed of a large number of interconnected processing units. It can imitate the information processing function of human brain neural system in different degrees and levels. Artificial neural network is a nonlinear and adaptive information processing system composed of a large number of interconnected processing units, which can imitate the information processing function of the human brain neural system in different degrees and levels and also can provide high accuracy automatic classification ability for signals with large amount of data and complex characteristics. Artificial neural network is mainly used in the field of information processing and pattern recognition. It is mainly used in the field of information processing and pattern recognition.

In the information fusion pattern recognition system, the information provided by each information source has certain uncertainty. Choosing the appropriate fusion algorithm is the core problem of the information fusion pattern recognition system. The process of fusion of information with uncertain features is essentially an uncertain reasoning process. Neural network has strong fault tolerance, selflearning, self-organization, and self-adaptive ability, can simulate complex nonlinear mapping, and can determine the classification standard according to the similarity of the samples accepted by the current system. The characteristics of neural network and its powerful nonlinear processing ability can meet the technical requirements of multi-sensor information fusion. In view of this, this paper uses BP neural network technology to study the intelligent recognition method of tool wear state.

\subsection{Structure Parameter Design of BP Neural Network}

4.1.1. Design of Input Layer and Output Layer. The energy values of P2 and P4 frequency bands of vibration signal, P4 frequency band of $\mathrm{AE}$ signal, and corresponding spindle speed are selected as the four main characteristic parameters of tool wear. Therefore, the input vector is determined to be 4 , that is, the number of neurons in the input layer is 4 . The output is the tool wear, so the number of neurons in the output layer is 1 .

4.1.2. Hidden Layer Design. According to Kolmogorov theorem, a three-layer BP neural network can realize the nonlinear mapping from any $n$ dimension to $m$ dimension. In this paper, a single hidden layer structure is selected. The number of nodes in the hidden layer directly affects the performance of neural network. The number of nodes is too small, and the pattern space is rough, which leads to poor fault tolerance and low ability of identifying the samples without learning. Too many nodes and too fine division of pattern space lead to too long network training time, slow or even nonconvergence of the network, and storage of the irregular content in the sample, reducing the generalization ability of the system. The number of hidden layer nodes is [26]

$$
l=\sqrt{n+m+a}
$$


where $l$ is the number of hidden layer nodes, $n$ is the number of input nodes, $m$ is the number of output nodes, and $a$ is the adjustment constant between 1 and 10 .

The BP neural network model is established among 4-15 hidden layer neurons by the trial method, and the optimal number of hidden layer neurons is determined by network error. When the number of hidden layer neurons is 9 , the network error is the smallest, and the number of hidden layer nodes is 9. Therefore, the 3-layer BP neural network structure based on the intelligent recognition of tool wear state based on information fusion is 4-9-1. According to the output of BP neural network, the wear degree of the tool can be directly determined.

4.2. Multi-Information Fusion Based on BP Neural Network. The physical quantities of vibration signal, acoustic emission signal, and spindle speed characteristic parameters have different meanings. Before neural network training, the characteristic data should be normalized. The normalization method was used.

$$
x_{i}^{0}=1-\frac{\left|x_{i}-\bar{x}\right|}{\left(x_{\max }-x_{\min }\right)} .
$$
$x_{i} / n$.

Among them, $\quad x_{\max }=\max \left\{x_{i}\right\}, x_{\min }=\left\{x_{i}\right\}, \bar{x}=\sum_{i=1}^{n}$

So, the values of the input vectors are all between 0 and 1 .

The hyperbolic tangent function is chosen as the activation function [27]. The initial weight of the network is randomly selected between $(0,1)$. The learning rate is 0.01 , the training error is $1 \times 10^{-8}$, and the maximum training period is 40000 . From the test characteristic data, 16 groups of $\mathrm{P} 2$ and $\mathrm{P} 4$ band energy value of the vibration signal and P4 band energy value of the acoustic emission signal are selected randomly, and the corresponding spindle speed also is selected together as the training samples to train the $\mathrm{BP}$ neural network. The 3-layer BP neural network model with 4-9-1 network structure is trained, and the training target precision is achieved through 31 steps. The training error curve is shown in Figure 8.

\section{Test Results and Analysis}

18 groups of $\mathrm{P} 2$ and $\mathrm{P} 4$ band energy value of the vibration signal and P4 band energy value of the acoustic emission signal which are not the training samples are selected from the test characteristic data, and the corresponding spindle speed also is selected as the test samples together. These test samples are input into the BP neural network to verify the intelligent recognition method of tool wear state.

Firstly, the pattern recognition method of support vector machine is used to recognize the tool wear state based on single sensor information. Then, BP neural network technology is used to identify the tool wear state by fusing the vibration signal, acoustic emission signal, and spindle speed feature information. Finally, the above discrimination results were compared with the actual detection results of tool wear $(18$ samples, including 1 initial

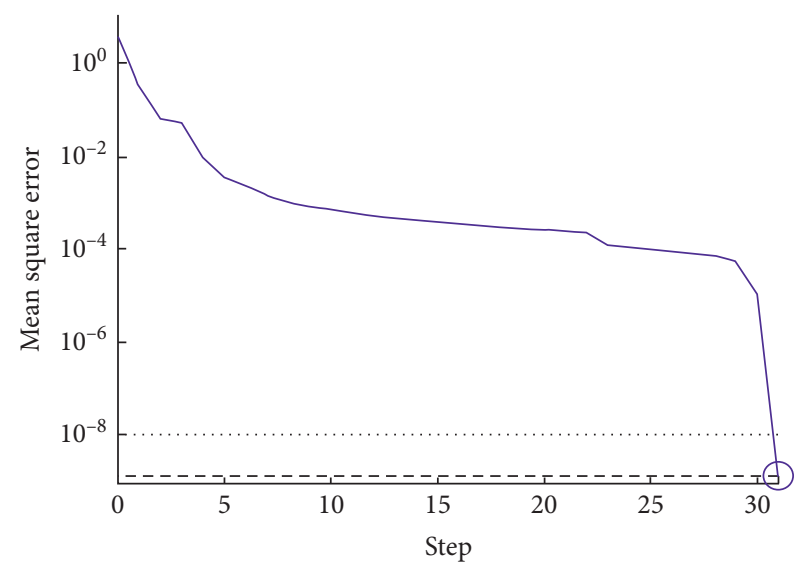

- Training
$\ldots--$ Optimal
$\ldots .$. Target

Figure 8: Training error curve.

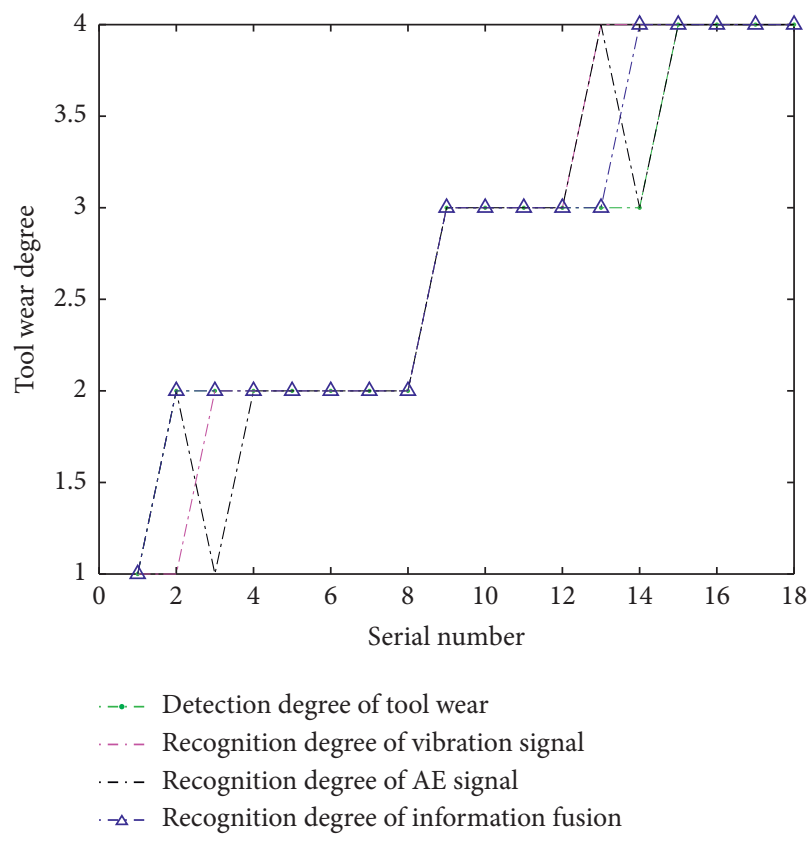

Figure 9: Tool wear detection and recognition degree.

wear, 7 types of normal wear, 6 types of moderate wear, and 4 types of severe wear).

The 18 samples were sorted according to the actual value of tool wear. The actual detection degree of tool wear, the recognition degrees by single vibration signal or single acoustic emission signal, and the recognition degree by information fusion are shown in Figure 9.

The results of single vibration signal identification were as follows: one normal wear (No. 1) was misjudged as initial wear, and two kinds of moderate wear (Nos. 13 and 14) were misjudged as severe wear. The recognition accuracy of all samples was $83.33 \%$. The results of single acoustic signal recognition were as follows: 1 normal wear (No. 3) was misjudged as initial wear, 1 medium wear (No. 13) was 


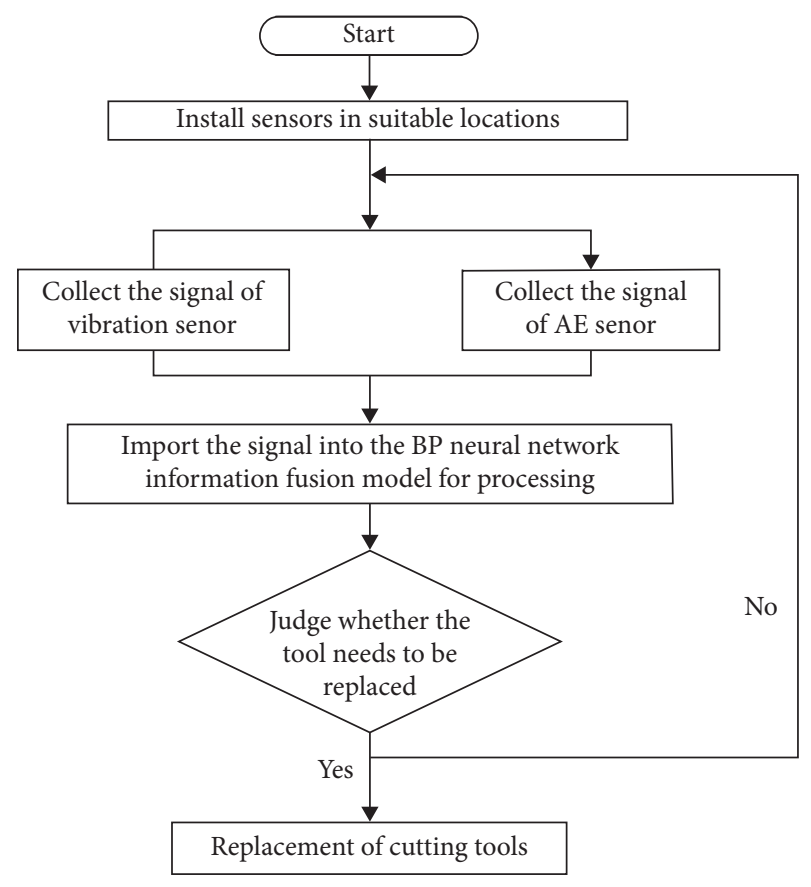

FIGURE 10: Diagnosis flowchart of tool wear status.

misjudged as severe wear, and the accuracy rate of identification for all samples was $88.89 \%$. The result of information fusion identification was as follows: one medium wear (No. 14) was misjudged as severe wear, and the accuracy of all samples was $94.44 \%$.

The accuracy of tool wear state recognition based on neural network multi-information fusion is $11.11 \%$ and $5.55 \%$ higher than that based on vibration signal and acoustic emission signal.

The diagnosis flowchart of the tool wear status is shown in Figure 10.

\section{Concluding Remarks}

Tool wear state directly affects the accuracy, efficiency, and cost of workpiece processing and the stability of the whole manufacturing equipment system.

(1) This paper studies the application of information fusion technology in tool wear state recognition. The multi-information data acquisition system of tool wear condition of $\mathrm{CNC}$ lathe is composed of a $\mathrm{CNC}$ lathe, workpiece, sensor, and signal amplifier and also includes a data acquisition, analysis, and processing device.

(2) In this paper, BP neural network is used to fuse the feature information of vibration signal and AE signal with high correlation with tool wear degree, a 3-layer BP neural network tool wear state intelligent recognition model with 4-9-1 structure is constructed, and the model is trained.

(3) The experimental results show that the recognition accuracy of the intelligent recognition system for tool wear state of CNC turning tool in all experimental samples is $94.44 \%$, which is $11.11 \%$ higher than that of vibration sensor and 5.55\% higher than that of $\mathrm{AE}$ sensor.

The accuracy of the tool wear state identified by the information fusion method is higher than that of the tool wear state identified by a single sensor, which effectively realizes the monitoring and automatic monitoring of the tool wear state and has a certain practical application value. However, the model established in this paper is only suitable for $\mathrm{CNC}$ lathe and has low universality.

\section{Data Availability}

The data used to support the findings of this study are included within the article.

\section{Conflicts of Interest}

The authors declare that they have no conflicts of interest.

\section{Acknowledgments}

The authors are grateful for the financial support provided by the National Natural Science Foundation of China under grant no. 51805151 and the Key Scientific Research Project of the University of Henan Province of China under grant no. $21 \mathrm{~B} 460004$.

\section{References}

[1] Q. Zhou and K. Liu, "Fault diagnosis of CNC machine tools common," Advanced Materials Research, vol. 317-319, pp. 1882-1885, 2011.

[2] X. Liao, G. Zhou, Z. Zhang, J. Lu, and J. Ma, "Tool wear state recognition based on GWO-SVM with feature selection of genetic algorithm," International Journal of Advanced Manufacturing Technology, vol. 104, no. 1, pp. 1051-1063, 2019.

[3] H. Yuan, S. Shudong, and L. Jingyao, "Prediction of cutting tool life based on ACO-BP neural network," Mechanical Science \& Technology for Aerospace Engineering, vol. 28, no. 11, pp. 1517-1521, 2009.

[4] Y. Hou, D. Zhang, B. Wu, and M. Luo, "Milling force modeling of worn tool and tool flank wear recognition in end milling," IEEE, vol. 20, no. 3, pp. 1024-1035, 2015.

[5] C. W. Xu, H. L. Chen, and Z. Liu, "State recognition technology and application on milling tool wear," Applied Mechanics and Materials, vol. 10-12, pp. 869-873, 2007.

[6] Z. Q. Li, P. Nie, and S. G. Zhao, "Identification method of tool wear based on locally linear embedding and support vector machine," Applied Mechanics and Materials, vol. 246-247, pp. 1289-1293, 2012.

[7] B. Ye, J. Liu, R. Peng, Y. Tang, and X. Zhao, "Study on fuzzy data fusion for real-time intelligent recognition of tool wear state," in Advances in Machining \& Manufacturing Technology IX School of Mechanical Engineering, South China University of Technology, Guangdong, China, 2008.

[8] G. Wang and X. Feng, "Tool wear state recognition based on linear chain conditional random field model," Engineering Applications of Artificial Intelligence, vol. 26, no. 4, pp. 1421-1427, 2013. 
[9] S. Guan, H. Pang, W. Song, and Z. Kang, "Cutting tool wear recognition based on MF-DFA feature and LS-SVM algorithm," Nongye Gongcheng Xuebao/Transactions of the Chinese Society of Agricultural Engineering, vol. 34, no. 14, pp. 61-68, 2018.

[10] K. Ge, L. Xu, X. Zhang et al., "Information fusion technology based on multi-sensor," International Core Journal of Engineering, vol. 6 , no. 8, 2020.

[11] T. Yan and X. Liu, "Fault diagnosis method of ship main machinery based on information fusion technology," Journal of Coastal Research, vol. 103, no. 1, pp. 905-908, 2020.

[12] S. Zhu, "Multi-source information fusion technology and its engineering application," Research in Health Science, vol. 4, no. 4 , p. $408,2020$.

[13] Y. Wang, "Information fusion technology based on multisensor," International Journal of Educational Management, vol. 4, no. 4, 2019.

[14] Z. Chen, "Multi-sensor information fusion technology and its application in target recognition," International Journal of New Developments in Engineering and Society, vol. 3, no. 5, 2019.

[15] M. Xiao, K. Li, W. Liu, and X. Xin, "Fast fault location method for power distribution systems based on multi-source information fusion technology," IOP Conference Series: Materials Science and Engineering, vol. 486, no. 1, Article ID 012070, 2019.

[16] T. Zhang, W. Ji, and Y. Qiu, "NC equipment fault diagnosis research in uncertain circumstance based on information fusion and bayesian networks integrated," IOP Conference Series: Materials Science and Engineering, vol. 493, no. 1, Article ID 012151, 2019.

[17] G. Li, Y. Wang, J. He, Q. Hao, H. Yang, and J. Wei, "Tool wear state recognition based on gradient boosting decision tree and hybrid classification RBM," International Journal of Advanced Manufacturing Technology, vol. 110, no. 1-2, 2020.

[18] Y. Du, G. Wu, Y. Tang, H. Cao, and S. Liu, "Reliability allocation method for remanufactured machine tools based on fuzzy evaluation importance and failure influence," International Journal of Precision Engineering and ManufacturingGreen Technology, 2020.

[19] A. Muzaffer, S. David, S. Max, A. Drik, and B. Thomas, "Kernel selection for support vector machines for system identification of a CNC machining center," IFAC-PapersOnLine, vol. 52, no. 29, pp. 192-198, 2019.

[20] G. Xu, H. Zhou, and J. Chen, "CNC internal data based incremental cost-sensitive support vector machine method for tool breakage monitoring in end milling," Engineering Applications of Artificial Intelligence, vol. 74, pp. 90-103, 2018.

[21] B. Jose, K. Nikita, T. Patil, S. Hemakumar, and P. Kuppan, "Online monitoring of tool wear and surface roughness by using acoustic and force sensors," Materials Today: Proceedings, vol. 5, no. 2, pp. 8299-8306, 2018.

[22] J. Herwan, S. Kano, O. Ryabov, H. Sawada, and M. Watanabe, "Comparing vibration sensor positions in CNC turning for a feasible application in smart manufacturing system," International Journal of Automation Technology, vol. 12, no. 3, pp. 282-289, 2018.

[23] T. Junge, H. Liborius, T. Mehner, A. Nestler, A. Schubert, and T. Lampke, "Measurement system based on the Seebeck effect for the determination of temperature and tool wear during turning of aluminum alloys," Procedia CIRP, vol. 93, pp. 1435-1441, 2020.
[24] R. Upase and N. Ambhore, "Experimental investigation of tool wear using vibration signals: an ANN approach," Materials Today: Proceedings, vol. 24, no. 2, pp. 1365-1375, 2020.

[25] Y. Liu, F. Wang, J. Lv, and X. Wang, "A novel method for tool identification and wear condition assessment based on multisensor data," Applied Sciences, vol. 10, no. 8, p. 2746, 2020.

[26] J. Michalkiewicz, "Modified Kolmogorov's neural network in the identification of Hammerstein and Wiener systems," IEEE transactions on neural networks and learning systems, vol. 23, no. 4, pp. 657-662, 2012.

[27] A. Ngaopitakkul and S. Bunjongjit, Selection of Proper Activation Functions in Back-Propagation Neural Network Algorithm for Transformer and Transmission System Protection, Springer, Berlin, Germany, 2015. 